Comment. Math. Helv. 74 (1999) 143-149

0010-2571/99/010143-7 \$1.50+0.20/0
(C) 1999 Birkhäuser Verlag, Basel

Commentarii Mathematici Helvetici

\title{
On the dilatation of extremal quasiconformal mappings of polygons
}

\author{
Kurt Strebel
}

\begin{abstract}
A polygon $P_{N}$ is the unit disk $\mathbb{D}$ with $n$ distinguished boundary points, $4 \leq n \leq N$. An extremal quasiconformal mapping $f_{0}: \mathbb{D}_{z} \rightarrow \mathbb{D}_{w}$ maps each polygon $P_{N}$ inscribed in $\mathbb{D}_{z}$ onto a polygon $P_{N}^{\prime}$ inscribed in $\mathbb{D}_{w}$. Let $f_{N}$ be the extremal quasiconformal mapping of $P_{N}$ onto $P_{N}^{\prime}$. Let $K_{N}$ be its dilatation and let $K_{0}$ be the maximal dilatation of $f_{0}$. Then, evidently $\sup K_{N} \leq K_{0}$. The problem is, when equality holds. This is completely answered, if $f_{0}$ does not have any essential boundary points. For quadrilaterals $Q$ and $Q^{\prime}=f_{0}(Q)$ the problem is $\sup \left(M^{\prime} / M\right)=K_{0}$, with $M$ and $M^{\prime}$ the moduli of $Q$ and $Q^{\prime}$ respectively.
\end{abstract}

Mathematics Subject Classification (1991). Primary 30C75; Secondary 30C62.

Keywords. Extremal qc mappings of disk; inscribed quadrilaterals and polygons.

\section{Introduction}

1. Let $h$ be a quasisymmetric mapping of the boundary of the unit disk $\mathbb{D}_{z}$ onto the boundary of $\mathbb{D}_{w}$ and let $f$ be a quasiconformal extension of $h$ into the disk. It is called extremal and denoted by $f_{0}$ if its maximal dilatation $K_{0}$ is smallest possible. We always assume $K_{0}>1$. The disk $\mathbb{D}_{z}$ becomes a quadrilateral $Q$ if we mark four different points $z_{j}, j=1, \ldots, 4$, in the positive direction on its boundary $\partial \mathbb{D}_{z}$. The mapping $f_{0}$ takes the vertices $z_{j}$ into points $w_{j}=f_{0}\left(z_{j}\right)$ on $\partial \mathbb{D}_{w}$ and thus the quadrilateral $Q$ into a quadrilateral $Q^{\prime}=f_{0}(Q)$ inscribed in $\mathbb{D}_{w}$. It follows from the definition of quasiconformality that the conformal moduli $M$ and $M^{\prime}$ of $Q$ and $Q^{\prime}$ respectively satisfy (for general properties of quasiconformal mappings, see [3])

$$
\frac{1}{K_{0}} M \leq M^{\prime} \leq K_{0} M
$$

It has been a question for some time, if the bound $K_{0}$ is best possible in the inequality (1), in other words, if the maximal dilatation $K_{0}$ of the extremal quasiconformal extension $f_{0}$ of $h$ can be determined by the ratio of the moduli of 
inscribed quadrilaterals,

$$
\sup \frac{M^{\prime}}{M}=K_{0} .
$$

The question has recently been answered in the negative by Anderson and Hinkkanen [1] by laborious computations of a counterexample (horizontal stretching of a parallelogram) and by Reich [4] who reduced it to an approximation problem for holomorphic functions. More counterexamples are given in [9].

2. It is easy to find examples where (2) holds; the above solutions consist therefore in the construction of examples where it does not hold. A type of the first kind is a vertical half strip $S$ and its horizontal stretching by $K_{0}$. Let $z=x+i y$, $S=\{z ; 0<x<a, 0<y\}, w=u+i v, S^{\prime}=\left\{w ; 0<u<K_{0} a, 0<v\right\}$. We make $S$ to a quadrilateral by marking the vertices $(0, a, a+i b, i b)$ for arbitrary $b>0$, and similarly $S^{\prime}$ by marking the image points $\left(0, K_{0} a, K_{0} a+i b, i b\right)$. Making use of the extremal length definition of the modulus of a quadrilateral ([3], p. 21) as the extremal distance of the vertical sides we easily find the estimates

$$
M \leq a / b, \quad M^{\prime} \geq K_{0} a /\left(b+K_{0} a\right)
$$

and thus

$$
K_{0} \geq \frac{M^{\prime}}{M} \geq \frac{K_{0} a}{b+K_{0} a} \cdot \frac{b}{a}
$$

which gives

$$
\lim _{b \rightarrow \infty} \frac{M^{\prime}}{M}=K_{0}
$$

3. The problem with the moduli of quadrilaterals has a different interpretation. We look at the extremal quasiconformal mapping $f$ of $Q$ onto $Q^{\prime}$. This is a mapping of $\mathbb{D}_{z}$ onto $\mathbb{D}_{w}$ which takes the vertices of $Q$ into those of $Q^{\prime}$. Its dilatation is $K=M^{\prime} / M$, and the question is now what happens with $K$ if we vary the vertices of $Q$ in all possible ways? Of course we always have $K \leq K_{0}$, but will we have $\sup K=K_{0}$ ? In this formulation the problem has a natural generalization to polygons, i.e. disks with an arbitrary finite number $n \geq 4$ of vertices. The basic extremal qc mapping $f_{0}$ assigns a polygon $P_{n}^{\prime}$ inscribed in $\mathbb{D}_{w}$ to each polygon $P_{n}$ inscribed in $\mathbb{D}_{z}$. The extremal qc mapping $f_{n}$ of $P_{n}$ onto $P_{n}^{\prime}$ (i.e. of course of $\mathbb{D}_{z}$ onto $\mathbb{D}_{w}$, but with the only requirement that the vertices of $P_{n}$ go into the vertices of $\left.P_{n}^{\prime}\right)$ is a Teichmüller mapping with a complex dilatation $\varkappa_{n}=k_{n}\left(\overline{\varphi_{n}} /\left|\varphi_{n}\right|\right)$, $k_{n}=\left(K_{n}-1\right) /\left(K_{n}+1\right)$. The quadratic differential $\varphi_{n}$ is rational, with at most first order poles at the vertices of $P_{n}$. Moreover, $\varphi_{n}(z) d z^{2}$ is real along the sides of $P_{n}$. Since $f_{0}$ also maps the vertices of $P_{n}$ onto those of $P_{n}^{\prime}$ and $f_{n}$ is extremal with this property, we have $K_{n} \leq K_{0}$. The question arises if, by varying the polygon $P_{n}$ in all possible ways, we have

$$
\sup K_{n}=K_{0} .
$$


4. It follows from general principles of qc mappings (we refer to [3] for the general theory) that this is in fact true if we allow the number $n$ of vertices to become arbitrarily large (for a proof see [5], p. 385, bottom). But how is it, if this number is bounded, $n \leq N$ say? With a certain natural restriction we will characterize the extremal mappings $f_{0}$ for which this happens. The proof is an application of the "polygon inequality" ([5], p. 384) and a theorem of R. Fehlmann ([2], p. 567).

\section{The polygon inequality}

5. Let $f_{0}$ be an extremal qc mapping of $\mathbb{D}_{z}$ onto $\mathbb{D}_{w}$ with $f_{0} \mid \partial \mathbb{D}_{z}=h$. Let $\varkappa_{0}$ with $\left\|\varkappa_{0}\right\|_{\infty}=k_{0}$ be its complex dilatation and $K_{0}=\left(1+k_{0}\right) /\left(1-k_{0}\right)$ its maximal dilatation. Mark $n$ points $z_{j}, j=1, \ldots, n$, on $\partial \mathbb{D}_{z}, 4 \leq n \leq N$. The disk $\mathbb{D}_{z}$ with the marked boundary points $z_{j}$ is called a polygon $P_{n}$. The image of $P_{n}$ by $f_{0}$ is the polygon $P_{n}^{\prime}$, inscribed in $\mathbb{D}_{w}$, with vertices $w_{j}=f_{0}\left(z_{j}\right)$. Let $f_{n}$ be the extremal qc mapping of $P_{n}$ onto $P_{n}^{\prime}, f_{n}\left(z_{j}\right)=w_{j}$, and let $\varphi_{n},\left\|\varphi_{n}\right\|=1$, denote the associated quadratic differential. The complex dilatation of $f_{n}$ is $k_{n}\left(\overline{\varphi_{n}} /\left|\varphi_{n}\right|\right)$. Then, the Polygon Inequality holds:

$$
\operatorname{Re} \iint_{|z|<1} \frac{\varkappa_{0}(z) \varphi_{n}(z)}{1-\left|\varkappa_{0}(z)\right|^{2}} d x d y \geq \frac{k_{n}}{1-k_{n}}-\iint_{|z|<1}\left|\varphi_{n}(z)\right| \frac{\left|\varkappa_{0}(z)\right|^{2}}{1-\left|\varkappa_{0}(z)\right|^{2}} d x d y .
$$

For the proof I refer to ([5], p. 384). In that paper, the inequality was used to prove that the "polygon differentials" $\varphi_{n}$ form a Hamilton sequence for $\varkappa_{0}$ if the number of vertices tends to infinity and the sides of the polygons $P_{n}$ become arbitrarily short. This led to a proof of the necessity of the Hamilton-Krushkal condition for extremality. Now, on the contrary, we restrict the number of vertices by a fixed number $N$, and we denote a polygon with $n \leq N$ vertices generically by $P_{N}$.

6.

Theorem 1. Let $f_{0}: \mathbb{D}_{z} \rightarrow \mathbb{D}_{w}$ with complex dilatation $\varkappa_{0}$, $\left\|\varkappa_{0}\right\|_{\infty}=k_{0}$, be extremal for its boundary values $h$. Assume that for a fixed number $N$ the polygon mappings $f_{N}: P_{N} \rightarrow P_{N}^{\prime}=f_{0}\left(P_{N}\right)$ with complex dilatation $k_{N}\left(\overline{\varphi_{N}} /\left|\varphi_{N}\right|\right)$ satisfy

$$
\sup k_{N}=k_{0}
$$

(This is of course equivalent to $\sup K_{N}=K_{0}$.) Then, there is a sequence of polygon mappings $f_{N}^{(i)}$ the quadratic differentials $\varphi_{N}^{(i)}$ of which, $\left\|\varphi_{N}^{(i)}\right\|=1$, form a Hamilton sequence for $\varkappa_{0}$, i.e.

$$
\operatorname{Re} \iint \varkappa_{0}(z) \varphi_{N}^{(i)}(z) d x d y \rightarrow k_{0}, \quad i \rightarrow \infty
$$


Proof. Assume first that $f_{0}$ has constant dilatation $\left|\varkappa_{0}(z)\right|=k_{0}$ a.e. Then, the polygon inequality yields

$$
\frac{1}{1-k_{0}^{2}} \operatorname{Re} \iint \varkappa_{0}(z) \varphi_{N}(z) d x d y \geq \frac{k_{N}}{1-k_{N}}-\frac{k_{0}^{2}}{1-k_{0}^{2}}
$$

for all polygons $P_{N}$. Let $P_{N}^{(i)}$ be a sequence of polygons the extremal mappings $f_{N}^{(i)}$ of which satisfy $k_{N}^{(i)} \rightarrow k_{0}$. Then

$$
\varliminf_{i \rightarrow \infty} \operatorname{Re} \iint \varkappa_{0}(z) \varphi_{N}^{(i)}(z) d x d y \geq \frac{k_{0}}{1-k_{0}}\left(1-k_{0}^{2}\right)-k_{0}^{2}=k_{0}
$$

On the other hand

$$
\operatorname{Re} \iint \varkappa_{0}(z) \varphi_{N}^{(i)}(z) d x d y \leq\left|\iint \varkappa_{0}(z) \varphi_{N}^{(i)}(z) d x d y\right| \leq k_{0} .
$$

This gives the result (9) in the case where $\left|\varkappa_{0}(z)\right|=k_{0}$ a.e. If $\left|\varkappa_{0}(z)\right|$ is not constant a.e. we proceed as in ([5], p. 386 and p. 382). However, in our present work we only need the case of constant $\left|\varkappa_{0}(z)\right|$.

Since the number of vertices of the polygons $P_{N}^{(i)}$ is smaller or equal to $N$, we can assume, by passing to a further subsequence, that they converge to a finite number $\leq N$ of points on $\partial \mathbb{D}_{z}$. We write $P_{N}^{(i)} \rightarrow P_{N}$.

The vertical half strip in the introduction is an example where the given quadrilaterals give rise to a Hamilton sequence for the horizontal stretching (which is uniquely extremal).

\section{Extremal mappings without essential boundary point}

7. Let $f_{0}$ with complex dilatation $\varkappa_{0},\left\|\varkappa_{0}\right\|_{\infty}=k_{0}$, be extremal for its boundary values $h$. A boundary point $z$ of $\mathbb{D}_{z}$ is called essential, if the following is true: For every neighborhood $U$ of $z$ and every qc mapping $g$ of $U \cap \mathbb{D}_{z}$ which is equal to $h$ on $U \cap \partial \mathbb{D}_{z}$ the maximal dilatation of $g$ is at least equal to $K_{0}=\left(1+k_{0}\right) /\left(1-k_{0}\right)$.

A theorem of R. Fehlmann ([2]), p. 567) says: If the complex dilatation $\varkappa_{0}$ has a degenerating Hamilton sequence (i.e. which tends to zero locally uniformly in the domain), then $f_{0}$ has an essential boundary point.

Combining this result with the considerations in ([7], p. 466) we can say: If $f_{0}$ does not have an essential boundary point, then, every Hamilton sequence for $\varkappa_{0}$ converges in norm to a holomorphic quadratic differential $\varphi_{0},\left\|\varphi_{0}\right\|=1$, and $\varkappa_{0}=k_{0}\left(\overline{\varphi_{0}} /\left|\varphi_{0}\right|\right)$ is the complex dilatation of $f_{0}$. 
8. Let us apply this to our case. Every polygon differential $\varphi_{N}^{(i)}$ can be continued across the boundary $\partial \mathbb{D}_{z}$ by reflection to a rational differential in the whole plane, of norm two. Therefore the limit $\varphi_{0}$ can be reflected. Since its norm is finite, it has at most first order poles at the $n \leq N$ limits of the vertices of the $P_{N}^{(i)}$, and $\varphi_{0}(z) d z^{2}$ is real along the subintervals of $\partial \mathbb{D}_{z}$ between these limits. Our main result is

Theorem 2. Let $f_{0}: \mathbb{D}_{z} \rightarrow \mathbb{D}_{w}$ be a qc mapping which is extremal for its boundary values, and assume that it does not have an essential boundary point. For fixed $N \geq 4$ denote the polygons with $4 \leq n \leq N$ vertices inscribed in $\mathbb{D}_{z}$ generically by $P_{N}$. To every $P_{N}$ the mapping $f_{0}$ determines a polygon $P_{N}^{\prime}$ inscribed in $\mathbb{D}_{w}$, simply by mapping the vertices of $P_{N}$ onto those of $P_{N}^{\prime}$. Assume that the extremal mappings $f_{N}: P_{N} \rightarrow P_{N}^{\prime}$ satisfy $\sup k_{N}=k_{0}$. Then, there is a convergent sequence $f_{N}^{(i)}$ of polygon mappings with $\varphi_{N}^{(i)} \rightarrow \varphi_{0}$ in norm, where $\varkappa_{0}=k_{0}\left(\overline{\varphi_{0}} /\left|\varphi_{0}\right|\right)$ is the complex dilatation of $f_{0} . f_{0}$ itself is the extremal qc mapping of a polygon with $n \leq N$ vertices, and every maximizing sequence $f_{N}^{(i)}, k_{N}^{(i)} \rightarrow k_{0}$, tends to $f_{0}$ uniformly, $\varphi_{N}^{(i)} \rightarrow \varphi_{0}$ in norm.

9. In order to see that the theorem is not empty, let $f: \mathbb{D}_{z} \rightarrow \mathbb{D}_{w}$ be an extremal polygon mapping and let $\varphi$ be the associated rational quadratic differential, $\varkappa=$ $k(\bar{\varphi} /|\varphi|)$ the complex dilatation. The vertices $z_{j}$ are either first order poles or regular points (i.e. $\varphi\left(z_{j}\right) \neq 0$ ) or zeroes of $\varphi$ of any order. Along the sides we have $\varphi(z) d z^{2}$ real, and thus the sides are composed of trajectories and orthogonal trajectories.

The first order poles and the zeroes are clearly the only candidates for an essential boundary point of $f$. In order to find the local maximal dilatation $H_{z}$ at such a point $z$ we first apply the mapping $\Phi=\int \sqrt{\varphi}$ and then the horizontal stretching by $K$. The integral $\Phi$ maps an interior half neighborhood of $z$ onto an angle with a horizontal and a vertical side. It is a right angle in the case of a first order pole and an angle which is a multiple of $\frac{1}{2} \pi$ in the case of a zero, possibly many sheeted. In the image $\mathbb{D}_{w}$ we have the same situation, with a quadratic differential $\psi$ and an integral $\Psi=\int \sqrt{\psi}$. The horizontal side of the angle is stretched by $K$ while the vertical side is mapped identically. It is known (and easy to see, using logarithms on both sides, see [6], p. 323) that the local extremal mapping with the given boundary values has dilatation $<K$. Since $f$ itself is extremal with dilatation $K$, it does not have any essential boundary point, thus satisfying our requirement.

10. Let now $f_{0}: P_{N} \rightarrow P_{N}^{\prime}$ with complex dilatation $\varkappa_{0}=k_{0}\left(\overline{\varphi_{0}} /\left|\varphi_{0}\right|\right)$ be an extremal polygon mapping. We can clearly take $f_{N}=f_{0}$ itself and get $\sup k_{N}=$ $k_{0}$. Actually we only need to consider the substantial boundary points of $f_{0}$ (= poles of $\left.\varphi_{0}\right)$, since the extremal mapping of the restricted polygon $\widetilde{P}_{N}$ onto $\widetilde{P}_{N}^{\prime}$ is 
the same as $f_{0}$.

Let $\widetilde{N}$ be the number of substantial boundary points of $f_{0}$. If, however, we only admit polygons with at most $N^{\prime} \leq \widetilde{N}-1$ vertices, we find $\sup k_{N^{\prime}}<k_{0}$. For, if $\sup k_{N^{\prime}}=k_{0}$ we would again arrive, by the same considerations as before, at an extremal polygon mapping $f_{N^{\prime}}$ with a quadratic differential $\varphi_{N^{\prime}}$ with at most $N^{\prime}$ first order poles, whereas $\varphi_{0}$ has $\widetilde{N}$ first order poles. Therefore $\varphi_{N^{\prime}} \neq \varphi_{0}$, a contradiction.

11. We started with the following question. Let $f_{0}$ with complex dilatation $\varkappa_{0}$, $\left\|\varkappa_{0}\right\|=k_{0}$, be a qc mapping of $\mathbb{D}_{z}$ onto $\mathbb{D}_{w}$ which is extremal for its boundary values and which does not have an essential boundary point. Inscribe quadrilaterals $Q$ into $\mathbb{D}_{z}$ and denote their images by $f_{0}$ in $\mathbb{D}_{w}$ by $Q^{\prime}$. The image $Q^{\prime}$ has, as its vertices, the images by $f_{0}$ of the vertices of $Q$. Let $M$ and $M^{\prime}$ be the moduli of $Q$ and $Q^{\prime}$ respectively. The question is, if (2) can hold.

Let $f$ with dilatation $K$ be the extremal mapping of $Q$ onto $Q^{\prime}$. The equation (2) is equivalent with

$$
\sup K=K_{0}
$$

where the sup is taken over all quadrilaterals $Q$. This is the special case of (8) for $N=4$. We find

Theorem 3. The extremal mapping $f_{0}$ satisfies (13) for the inscribed quadrilaterals $Q$ if and only if it is the extremal mapping of a quadrilateral itself.

This means that in all other cases we have inequality in (13). The example of Anderson and Hinkkanen is the horizontal stretching of a parallelogram. This mapping $f_{0}$ has no essential boundary point and is, in their situation, not the mapping of quadrilaterals. Therefore $\sup \left(M^{\prime} / M\right)<K_{0}$.

The example of Reich has analytic boundary values. Therefore we have again $\sup \left(M^{\prime} / M\right)<K_{0}$.

Clearly, in both examples, we still have inequality in (13) even if we allow any inscribed polygons with an arbitrary fixed bound $N$ for the number of vertices.

Added in Proof. After the completion of this paper I have become aware of two papers with related results: Shanshuang Yang, On dilatations and substantial boundary points of homeomorphisms of Jordan curves, Results Math. 31 (1979), 180-188, and Qi Yi, A problem in extremal quasiconformal extensions, Sci. China Ser. A 41:11 (1998), 1135-1141.

\section{References}

[1] J. M. Anderson and A. Hinkkanen, Quadrilaterals and extremal quasiconformal extensions, Comment. Math. Helv. 70 (1995), 455-474. 
[2] R. Fehlmann, Über extremale quasikonforme Abbildungen, Comment. Math. Helv. 56 (1981), 558-580.

[3] O. Lehto and K. Virtanen, Quasiconformal Mappings in the Plane, Springer-Verlag, 1973, pp. $1-258$.

[4] E. Reich, An approximation condition and extremal quasiconformal extensions, Proc. Amer. Math. Soc. 125(5) (1997), 1479-1481.

[5] E. Reich and K. Strebel, Extremal quasiconformal mappings with given boundary values, in: Contributions to Analysis, Academic Press, 1974, pp. 375-391.

[6] K. Strebel, Zur Frage der Eindeutigkeit extremaler quasikonformer Abbildungen des Einheitskreises, Comment. Math. Helv. 36 (1962), 306-323.

[7] K. Strebel, On the existence of extremal Teichmueller mappings, J. Analyse Math. 30 (1976), 464-480.

[8] K. Strebel, Quadratic Differentials, Ergeb. Math. Grenzgeb. 3:5, Springer-Verlag, 1984, pp. $1-184$.

[9] S. Wu, Moduli of quadrilaterals and extremal quasiconformal extensions of quasisymmetric functions, Comment. Math. Helv. 72(4) (1997).

Kurt Strebel

Freiestraße 14

CH-8032 Zürich

Switzerland

e-mail: kstrebel@math.unizh.ch

(Received: December 23, 1997) 